\title{
Longitudinal analysis of blood markers reveals progressive loss of resilience and predicts ultimate limit of human lifespan
}

\author{
Timothy V. Pyrkov ${ }^{a, *}$, Konstantin Avchaciov $^{a}$, Andrei E. Tarkhov ${ }^{a, b}$, \\ Leonid I. Menshikov ${ }^{a, c}$, Andrei V. Gudkov ${ }^{d, e}$ and Peter O. Fedichev ${ }^{a, f}$ \\ ${ }^{a}$ Gero LLC, 105064, Nizhny Susalny per. 5/4, Moscow, Russia \\ ${ }^{b}$ Skolkovo Institute of Science and Technology, Bolshoy Boulevard 30, bld. 1, Moscow 121205, Russia \\ ${ }^{c}$ National Research Center "Kurchatov Institute", 1, \\ Akademika Kurchatova pl., Moscow 123182, Russia \\ ${ }^{d}$ Roswell Park Comprehensive Cancer Center, Elm and Carlton streets, Buffalo, NY 14263, USA \\ ${ }^{e}$ Genome Protection, Inc. 640 Ellicott St \#444, Buffalo, NY 14203, USA and \\ ${ }^{f}$ Moscow Institute of Physics and Technology, 141700, \\ Institutskii per. 9, Dolgoprudny, Moscow Region, Russi£*
}

\begin{abstract}
We analyzed aging trajectories of complete blood counts (CBC) and their association with the incidence of chronic diseases and death in cohorts of aging individuals registered in the UK Biobank and National Health and Nutrition Examination Survey (NHANES) studies. Application of a proportional hazards model to the CBC data allowed us to identify the log-transformed hazard ratio as a quantitative measure of aging process, which we have named the dynamic morbidity index (DMI). DMI increased with age in the UK Biobank and NHANES cohorts, was associated with multiple morbidity, and predicted the prospective incidence of age-related diseases and death. To better understand the nature of DMI variations along individual aging trajectories, we acquired a large longitudinal database of CBC measurements from a consumer diagnostics laboratory. We observed that population DMI distribution broadening can be explained by a progressive loss of physiological resilience measured by the DMI auto-correlation time. Extrapolation of this data suggested that DMI recovery time and variance would simultaneously diverge at a critical point of 120-150 years of age corresponding to a complete loss of resilience. We conclude that the criticality resulting in the end of life is an intrinsic biological property of an organism that is independent of stress factors and signifies a fundamental or absolute limit of human lifespan.
\end{abstract}

\section{INTRODUCTION}

Aging is manifested as a progressive functional decline leading to exponentially increasing prevalence [1, 2] and incidence of chronic age-related diseases (e.g., cancers, diabetes, cardiovascular diseases, etc. [3] [5] and diseasespecific mortality [6]. Much of our current understanding of the relationship between aging and changes in physiological variables over an organism's lifespan originates from large cross-sectional studies, including the National Health and Nutrition Examination Survey (NHANES; 40592 subjects, age range $18-85$ y.o.) and UK Biobank (UKB; 471473 subjects, age range $39-73$ y.o.) studies. Analysis of such large datasets has identified a number of "biological clocks", including those reflecting agerelated variations in blood markers [7, DNA methylation (DNAm) states [8, 9] or patterns of locomotor activity 10 12. Typically, the physiological indices change from the levels observed in the young organism at a much lower pace than it could be from Gompertz mortality law. Most important factors that are strongly associated with age are also known as the hallmarks of aging [13] and may be, at least in principle, modified pharmacologically. Hence,

\footnotetext{
* To whom correspondence should be addressed. E-mail: tim.pyrkov@gero.com, peter.fedichev@gero.com
}

an improved understanding of the relationship between the linear physiological state dynamics and the exponentially increasing morbidity and mortality observed during aging is needed to facilitate the rational design, development, and clinical validation of anti-aging interventions.

In this paper, we addressed these theoretical and practical issues by a systematic investigation of aging, organism state fluctuations, and gradual loss of resilience in a large dataset involving multiple Complete Blood Counts (CBC) measurements from the same individual. Following [14, 15], we built a biomarker of aging in the form of the log-mortality risk predictor from $\mathrm{CBC}$ variables. First, we characterized its association with age, multiple morbidity, lifestyle, and its prognostic performance for mortality and future morbidity risks assessment. Then, we observed that the dynamics of the biomarker of age along the individual aging trajectories is consistent with that of a stochastic process (random walk) on top of the slow aging drift. The increase in the biomarker variability is approximately linear with age and can be explained by the rise of the biomarker's auto-correlation time. The auto-correlation time is thus an independent biomarker of aging that is characteristic of resilience since it quantifies the organism state rigidity in response to stresses (such as the recovery time characterizing a transition from a nonchronic illness back to the healthy state). Our analysis shows that the auto-correlation time grows with age from 
about 2 weeks to over 4 weeks for cohorts aging from 40 to 70 years. We put forward arguments suggesting that the progressive loss of resilience with age may be a dynamic origin of the Gompertz law. Finally, we noted, by extrapolation, that the recovery time would diverge and hence the resilience would be ultimately lost at the critical point at the age in the range of $120-150$ years, thus indicating the absolute limit of human lifespan.

\section{RESULTS}

\section{Quantification of aging based on complete blood counts (CBC) measurements}

Complete blood count $(\mathrm{CBC})$ measurements are included in standard blood tests and comprise the largest common subset of data available for both the NHANES and UKB study populations (see Table S1). In investigating whether CBC dynamics could be used to quantify aging, we found that co-clustering of age- and sex-adjusted CBC components revealed two dynamic subsystems associated with oxygenation and immune functions, represented by variations in red blood cell counts as well as total and mean corpuscular hemoglobin levels, and with platelet and white blood cell counts, respectively (Fig. 1A.

Since rates of morbidity and mortality increase exponentially with age, a log-linear risk model is a good starting point for quantification of the aging process [12, 14]. We used the death register of the NHANES study (3792 death events observed in the follow-up by year 2015) and trained the Cox proportional hazards model [16] using the CBC measurements and sex variables (but not age) from 23807 study participants aged 40 y.o. and older. We therefore assumed that the risk of death depended on the organism state at the time of CBC measurement and the follow-up time only. The model yielded a loghazards ratio (log-HR) estimate (a linear combination of log-transformed CBC variables) for every participant. After adjustment for sex and age, this predictor was demonstrated to be equally well associated with mortality in the NHANES study $(\mathrm{HR}=1.43)$ and in the independent UKB study $(\mathrm{HR}=1.39$; Table S2), which was used as a validation dataset.

For the NHANES cohort, the CBC log-HR of the Cox mortality model gradually increased with age (Pearson's correlation coefficient $\mathrm{r}=0.29, \mathrm{p}<10^{-100}$, Fig. 1B. This parameter changed at a comparable rate in the independent UKB cohort; however, the correlation coefficient was lower in this cohort due to the more limited age range of the subjects $\left(\mathrm{r}=0.13, \mathrm{p}<10^{-100}\right.$, Fig. S1A .

To differentiate between the effects of chronic diseases and disease-free aging, we followed [17] and characterized the health status of each study participant based on their diagnosis with health conditions considered to be the most prevalent age-related conditions. The number of health conditions diagnosed for an individual was nor- malized to the total number of conditions included in the analysis to yield the "compound morbidity index" (CMI) with values ranging from zero to one. The list of health conditions common to the NHANES and UKB studies that were used for CMI determination is given in Table S2 and described in SI Appendix.

The association between multiple morbidity and estimated mortality risk is readily seen as the difference between the solid and dashed lines in Fig. $1 \mathrm{~B}$, which represent the mean log-HR values in the cohorts of healthy ("non-frail", CMI < 0.1) and "most frail" (CMI > 0.6) NHANES participants, respectively. Given the association between all-cause mortality risk and morbidity, we propose to refer to the log-HR of the mortality model as the "dynamic morbidity index" (DMI).

In groups stratified by increasing number of health condition diagnoses, the normalized distribution of DMI (after adjustment by mean DMI in age- and sex-matched cohorts of healthy subjects) exhibited a progressive shift to higher risk values and more variability (Fig. $1 \mathrm{C}$ for NHANES, Fig. S1B for UKB). For both NHANES and UKB, the largest shift was observed in the "most frail" $(\mathrm{CMI}>0.6)$ population. The increasingly heavy tail at the high end of the DMI distribution in this group is characteristic of an admixture of a distinct group of individuals occupying the adjacent region in the configuration space corresponding to the increased DMI variable. Therefore, DMI displacement after adjustment for age and sex in cohorts of healthy subjects was expected to be determined by the fraction of "most frail" individuals in a cohort of any given age. This was confirmed to be true using the NHANES dataset (Fig. 2A $\mathrm{r}=0.83$, $\left.\mathrm{p}=8.8 \times 10^{-9}\right)$.

The fraction of "most frail" subjects still alive increased exponentially at every given age until the age corresponding to the end of healthspan was reached. The characteristic doubling rate constants for the "most frail" population fractions were 0.08 and 0.10 per year in the NHANES and the UKB cohorts, respectively, in comfortable agreement with the accepted Gompertz mortality doubling rate of 0.085 per year [18, see Fig. 2B]

We note that the prevalence of diseases in the NHANES cohort is consistently higher than that in the UKB population, in spite of the lack of comparable lifespan in the two countries. This may be a consequence of the enrollment bias in the UKB: according to comparison with life tables in [19, the UKB subjects appear to live longer than typical UK residents.

\section{Dynamic morbidity index and health risks}

In the most healthy subjects, i.e. those with no diagnosed diseases at the time of assessment, the DMI predicted the future incidence of chronic age-related diseases observed during 10-year follow-up in the UKB study (Table $\mathrm{S2}$. There was no relevant information available in NHANES. We tested this association using a series of Cox 


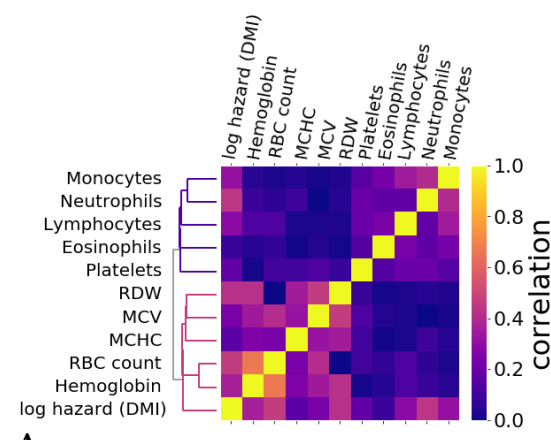

A

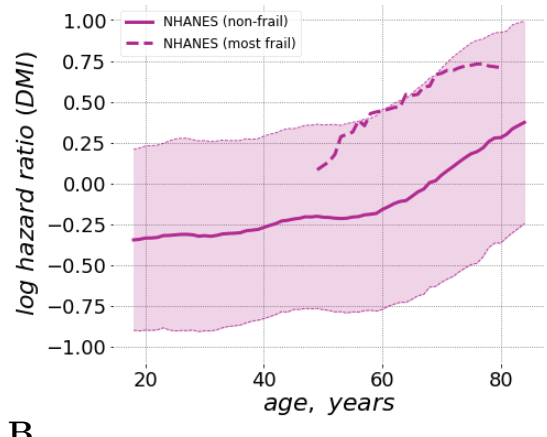

B

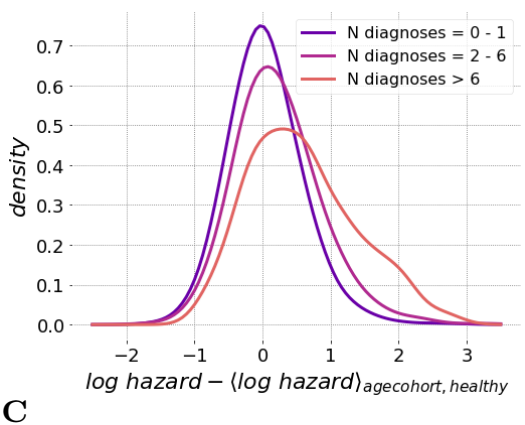

C

FIG. 1: A. Co-clustering of the age- and sex-adjusted CBC feature fluctuations in the NHANES dataset revealed two functionally related blood cell types, generally representing immune and oxygenation functions. We also included the dynamic morbidity index (DMI), the log-hazard ratio of a mortality risk model trained using the NHANES samples. As indicated by the vertical bar along the right-hand edge of the figure, the colors represent the absolute values of the Pearson's correlation coefficients between features. B. DMI mean values (solid line) and variance (shaded area) are plotted relative to age for the "non-frail" (combined morbidity index, CMI < 0.1) participants of NHANES study. The average DMI of the "most frail" (CMI > 0.6) individuals is shown with the dashed line. Data for other datasets investigated in this study are given in Supplementary Information (Fig. S1A). C. Distributions of sex- and age-adjusted DMI in cohorts of NHANES participants in different morbidity categories relative to the DMI mean in cohorts of "non-frail" ( 1 or no diagnoses, CMI $<0.1)$ individuals. Note that the distribution function in the "most frail" group (more than 6 diagnoses, $\mathrm{CMI}>0.6$ ) exhibited the largest shift and a profound deviation from the symmetric form.

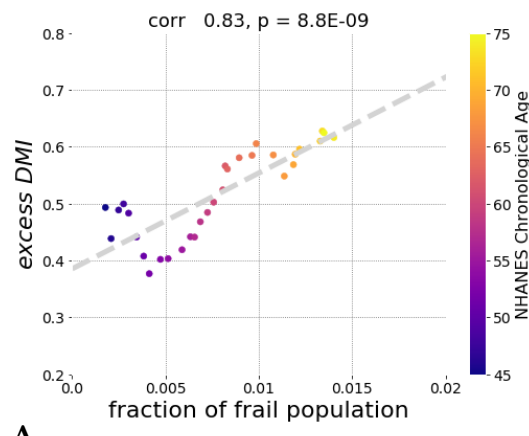

A

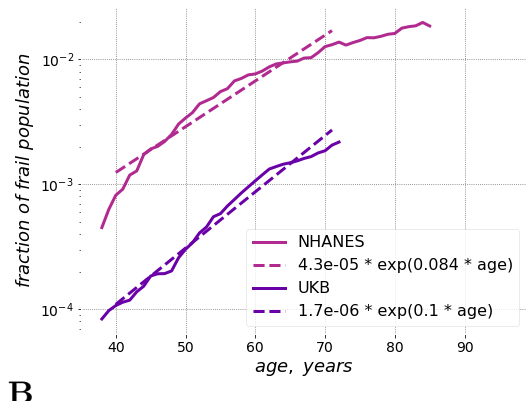

B

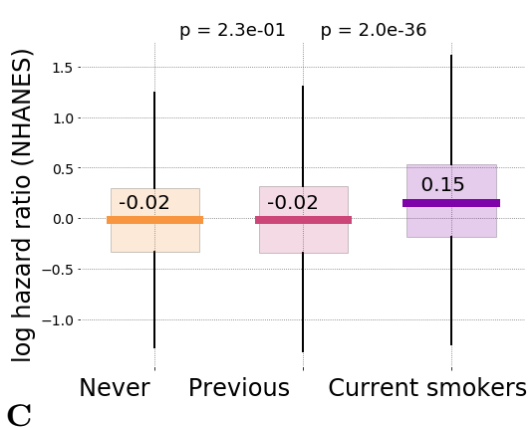

C

FIG. 2: A. Fraction of frail persons is strongly correlated with the excess DMI, that is the difference between the DMI of an individual and its average and the sex- and age-matched cohort in the "non-frail" population in NHANES. B, Exponential fit showed that until the age of 70 y.o. the fraction of the "most frail" individuals in the population grows approximately exponentially with age with the doubling rate constants of 0.08 and 0.10 per year in the NHANES and the UKB cohorts, respectively. C. Distribution of log hazards ratio in age- and sex-matched cohorts of NHANES participants who never smoked, smoked previously but quit prior to the time of study participation, or were current smokers at the time of the study.

proportional hazard models trained to predict the age at the onset/diagnosis of specific diseases. We observed that the morbidity hazard ratios associated with the DMI relative to its mean in age- and sex-matched cohorts were statistically significant predictors for at least the most prevalent health conditions (those with more than 3000 occurrences in the UKB population). The effect size $(\mathrm{HR} \approx 1.03-1.07)$ was the same regardless of whether a disease was diagnosed first in a given individual or followed any number of other diseases. Only emphysema and heart failure which are known to be strongly associated with increased neutrophill counts [20, 21] demon- strated particularly high associations. Therefore, we conclude that the DMI is a characteristic of overall health status that is universally associated with the risks of developing the most prevalent diseases and, therefore, with the end of healthspan as indicated by the onset of the first morbidity ( $\mathrm{HR} \approx 1.05$ for the "First morbidity" entry in Table $\mathrm{S} 2$.

In "non-frail" individuals with life-shortening lifestyles/behaviors, such as smoking, the DMI was also elevated, indicating a higher level of risks of future diseases and death (Fig. 2C). Notably, however, this effect appeared to be reversible: while the age- and sex- 
bioRxiv preprint doi: https://doi.org/10.1101/618876; this version posted February 7, 2020. The copyright holder for this preprint (which was not certified by peer review) is the author/funder. All rights reserved. No reuse allowed without permission.

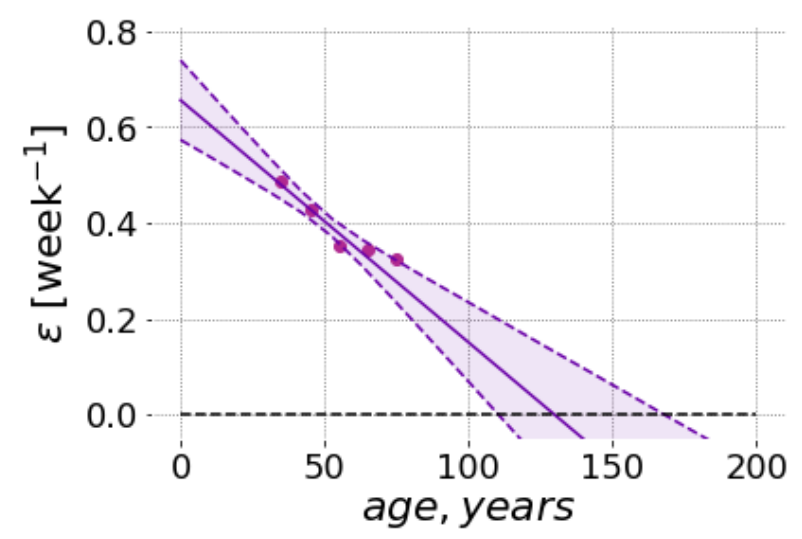

A

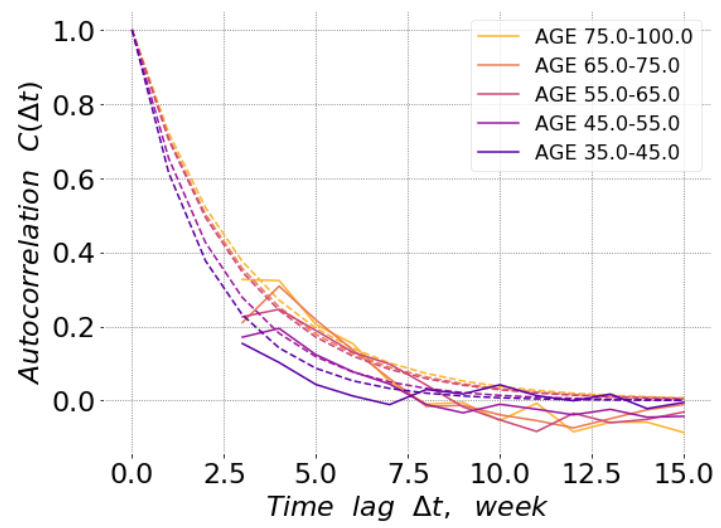

B
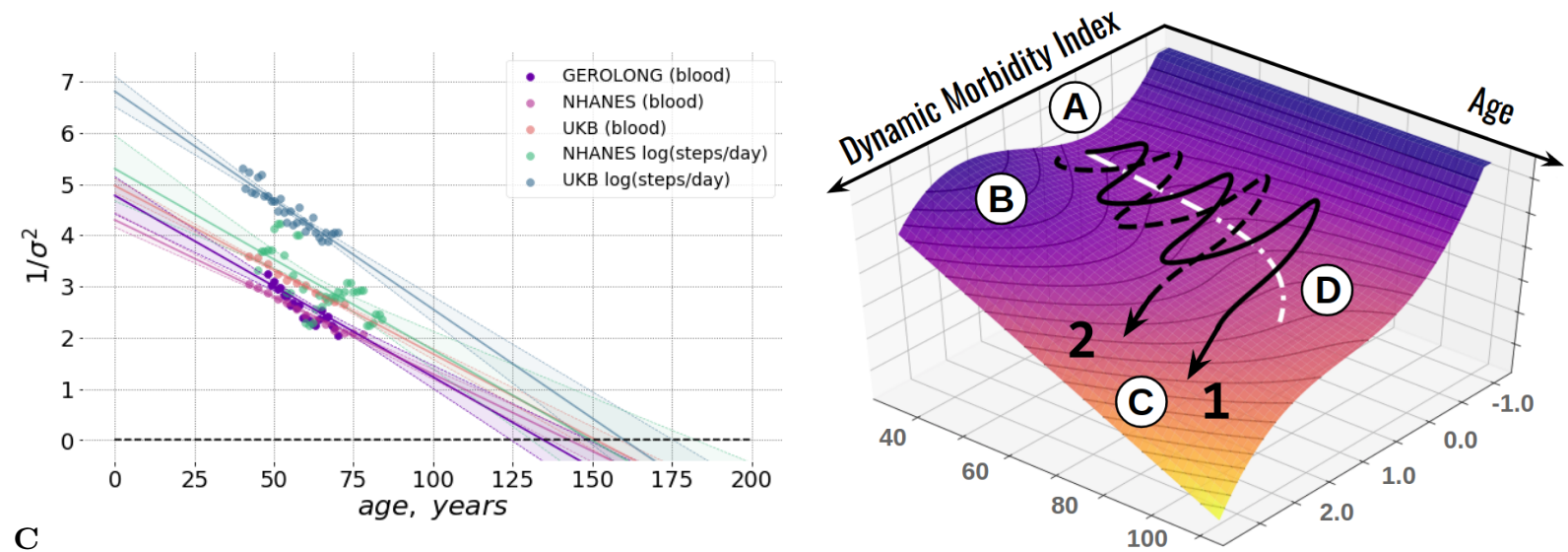

D

FIG. 3: A. The DMI relaxation rate (or the inverse characteristic recovery time) computed for sequential age-matched cohorts from the GEROLONG dataset decreased approximately linearly with age and could be extrapolated to zero at an age in the range of $\sim 110-170$ y.o. (at this point, there is complete loss of resilience and, hence, loss of stability of the organism state). The shaded area shows the $95 \%$ confidence interval. B. The auto-correlation function $C(\Delta t)$ of the DMI fluctuations during several weeks averaged in sequential 10-year age-cohorts of GEROLONG subjects showed gradual age-related remodelling. Experimental data and fit to autocorrelation function are shown with solid and dashed lines, respectively (see details in Supplementary Information). The DMI correlations are lost over time $\Delta t$ between the measurements and, hence, the DMI deviations from its age norm reach the equilibrium distribution faster in younger individuals. C. The inverse variance of DMI decreased linearly in all three investigated datasets and its extrapolated value vanished (hence, the variance diverged) at an age in the range of $120-150$ y.o. We performed the linear fit for subjects 40 y.o. and older, excluding the "most frail" (CMI >0.6) individuals. The shaded areas correspond to the $95 \%$ confidence intervals. The blue and green dots and lines show the inverse variance of log-scaled measure of total physical activity (the number of steps per day recorded by a wearable accelerometer) for UKB and NHANES participants, respectively. D. Representative aging trajectories are superimposed over the potential energy landscape (vertical axis) representing regulatory constraints. The stability basin "A" is separated from the unstable region "C" by the potential energy barrier "B". Aging leads to a gradual decrease in the activation energy and barrier curvature and an exponential increase in the probability of barrier crossing. The stochastic activation into a dynamically unstable (frail) state is associated with acquisition of multiple morbidities and certain death of an organism. The white dotted line "D" represents the trajectory of the attraction basin minimum. Examples 1 (black solid line) and 2 (black dashed line) represent individual life-long stochastic DMI trajectories that differ with respect to the age of first chronic disease diagnosis.

adjusted DMI means were higher in current smokers compared to non-smokers, they were indistinguishable between groups of individuals who never smoked and who quit smoking (c.f. [12, 22]).

\section{Physiological state fluctuations and loss of resilience}

To understand the dynamic properties of the organism state fluctuations in relation to aging and diseases, we acquired a large anonymized set of longitudinal CBC 
measurements from InVitro, the major Russian clinical diagnostics laboratory. This dataset, which we referred to as GEROLONG, included 828 male and 1517 female subjects aged $35-90$ with complete CBC analyses that were sampled $12-20$ times within a period of up to 42 months.

Consistently with our previous observations in the NHANES and UKB cohorts, DMI also increased with age in the longitudinal GEROLONG cohort. The average DMI value and its population variance at any given age were, however, considerably larger than those in the reference "non-frail" groups from the NHANES and UKB studies (see Fig. S1A). This difference likely reflects an enrollment bias: many of the GEROLONG blood samples were obtained from patients visiting clinic centers, presumably due to health issues. This could explain why the GEROLONG population appeared generally more frail in terms of DMI than the reference cohorts of the same age from other studies (Fig. S1A, compare the relative positions of the solid blue line and the two dashed lines representing the GEROLONG cohort and the frail cohorts of the NHANES and UKB studies, respectively). There was no medical condition information available for the GEROLONG subjects. Hence, we used the mean DMI of the "most frail" NHANES and UKB participants (which coincided, approximately, with the mean DMI of all GEROLONG subjects) as the cutoff value to select "non-frail" GEROLONG individuals.

Within the "non-frail" GEROLONG population (274 male and 682 female subjects aged $35-90$ ), serial CBC measurements along the individual aging trajectories over periods of time of up to three years revealed large stochastic fluctuations of the DMI around its mean values, which were considerably different among individual study participants. The averaged DMI auto-correlation function (see e.g., [23]) decayed exponentially as a function of the time delay between measurements within approximately a month (see Fig. 3B).

The inverse auto-correlation time is the basic statistical property of a stationary stochastic process and is an indicator of the relaxation (recovery) rate, characterizing the time scale involved in the equilibration of a system's state in response to external perturbations (see SI Appendix). We therefore propose using this quantity as a measure of an organism's "resilience", the capacity of an individual organism to resist and recover from the effects of physiological or pathological stresses [24, 25]).

We fitted the auto-correlation functions to an exponential function of the time delay and observed that recovery rates obtained from fitting to data in the subsequent age-cohorts decreased approximately linearly with age (Fig 3A). Extrapolation to older ages suggested that the equilibration rate vanishes and, hence, the recovery time becomes formally infinite, at an age of approximately 120 - 150 y.o. (95\% CI, 110 - 170 y.o.).

The variance of DMI increased with age in every dataset evaluated in this study. Since the dynamic range of random fluctuations would be inversely proportional to the recovery force (or the recovery rate, see [23]), we plotted the inverse variance of the DMI computed in sex- and age-matched cohorts of healthy persons (Fig. 3C). Again, extrapolation suggested that, if the tendency holds at older ages, the population variability would increase indefinitely at an age of approximately $120-150$ y.o.

The amplification of the fluctuations of the organism state variables with age is not limited to CBC features. In [12] we observed, that the variability of physical activity (namely, the logarithm of the average physical activity), that is another hallmark of aging and is associated with age and risks of death or major deceases, also increases with age. In Fig. $3 \mathrm{C}$ we plotted the inverse variance of this physical activity feature and found that it linearly decreases with age in such a way that the corresponding variance diverges at the same critical point at the age of approximately $120-150$ y.o.

\section{DISCUSSION}

We investigated aging trajectories of human CBC values and their association with risks of chronic age-related diseases, mortality and life-shortening lifestyles. We produced a proportional hazards mortality model using NHANES dataset and defined its log-hazard ratio prediction as the dynamic morbidity index (DMI). This quantitative parameter displayed all of the expected properties of a biomarker of aging in several large independent datasets: DMI increased with age, was predictive of the prospective incidence of age-related diseases and death, and was associated with typical life-shortening lifestyles, such as smoking, and morbidity. These findings support the idea that predictors from log-linear mortality or morbidity risk models can be effectively used to quantify the progress of aging and effects of lifestyles and diseases [12, 14, 26].

The simultaneous divergence of the organism state recovery times (critical slowing down) and the range of DMI variations (critical fluctuations) is characteristic of proximity of a critical point [23, 27] at some advanced age over 100 y.o. Under these circumstances, the organism state dynamics are stochastic and dominated by the variation of the single dynamic variable associated with the criticality, the DMI (Fig. 3D. Schematically, far from the critical point (at younger ages), the organism state perturbations can be thought of as confined to the vicinity of a possible stable equilibrium state in a potential energy basin $(A)$. Initially, the dynamic stability is provided by a sufficiently high potential energy barrier $(B)$ separating this stability basin from the inevitably present dynamically unstable regions $(C)$ in the space of physiological parameters. While in stability basin, an organism follows the trajectory $(D)$ of the equilibrium state, which is gradually displaced with aging even for the successfully aging individuals.

The DMI auto-correlation times (one-two months, see 
Fig. 3B are much shorter than lifespan. The dramatic separation of time scales makes it very unlikely that the linear decline of the recovery force measured by the recovery rate in Fig. $3 \mathrm{~A}$ can be explained by the dynamics of the organism state captured by the DMI variation alone. Therefore, we conclude that the progressive remodeling of the attraction basin geometry reflects adjustment of the DMI fluctuations to the slow independent process that is aging itself. In this view, the aging drift of the DMI mean in cohorts of healthy individuals (as in Fig. 1B) is the adaptive organism-level response to ever increasing stress produced by the aging process.

The longitudinal analysis in this work demonstrated that the DMI undergoes a random walk under the influence of stochastic stress factors and slowly increases, on average, as the organism ages. The dynamic range of the DMI fluctuations is inversely proportional to the recovery rate of the DMI fluctuations, and hence the two parameters are dependent quantities. Therefore, the mean DMI, on the one hand, and the dependent variables, the DMI variability and the auto-correlation time, on the other, comprise the minimum pair of independent biomarkers of human aging.

The DMI recovery rate characterizes fluctuations of DMI on time scales of a few months or more, decreases with age and thus indicates the progressive loss of physiological resilience. Such age-related remodeling of recovery rates has been previously observed in studies of various physiological and functional parameters in humans and other mammals. For example, in humans, a gradual increase in recovery time required after macular surgery was reported in sequential 10-year age cohorts [28] and age was shown to be a significant factor for twelve months recovery and the duration of hospitalization after hip fracture surgery [29, 30], coronary artery bypass [31], acute lateral ankle ligament sprain [32]. A mouse model suggested that the rate of healing of skin wounds also can be a predictor of longevity [33. This age-related slowing down of recovery rate may be independent of an organism ability to resist to stress [34. Recovery rate has been shown as a predictor of mortality on its own 35] and thus may serve as an early sign of health condition outcomes [27, 36. By analogy to resilience in ecological systems, the concept of physical resilience measured as the rate of recovery has been introduced in recent reviews [37, 38.

The resilience can only be measured directly from high-quality longitudinal physiological data. Framingham Heart Study [39], Dunedin Multidisciplinary Health and Development Study [40] and other efforts produced a growing number of reports involving statistical analysis of repeated measurements from the same persons, see, e.g., [41, 42. Most of the time, however, the subsequent samples are years apart and hence time between the measurements greatly exceeds the organism state autocorrelation time reported here. This is why, to the best of our understanding, the relation of the organism state recovery rate and mortality has remained largely elusive.
In the presence of stresses, the loss of resilience should lead to destabilization of the organism state. Indeed, in a reasonably smooth potential energy landscape forming the basin of attraction, the activation energy required for crossing the protective barrier $(B)$ decreases along with the curvature at the same pace, that is, linearly with age. Whenever the protective barrier is crossed, dynamic stability is lost (see example trajectories 1 and 2 in Fig. 3D which differ by the age of crossing) and deviations in the physiological parameters develop beyond control, leading to multiple morbidities, and, eventually, death.

On a population level, activation into such a frail state is driven by stochastic forces and occurs approximately at the age corresponding to the end of healthspan, understood as "disease-free survival". Since the probability of barrier crossing is an exponential function of the required activation energy (i.e., the barrier height) 23, the weak coupling between DMI fluctuations and aging is then the dynamic origin of the Gompertz mortality law. Since the remaining lifespan of an individual in the frail state is short, the proportion of frail subjects at any given age is proportional to the barrier crossing rate, which is an exponential function of age (see Fig. 2B).

The end of healthspan can therefore be viewed as a form of a nucleation transition [23], corresponding in our case to the spontaneous formation of states corresponding to chronic diseases out of the metastable phase corresponding to healthy organisms. The DMI is then the order parameter associated with the organism-level stress responses at younger ages and plays the role of the "reaction coordinate" of the transition to the frail state later in life. All chronic diseases and death in our model originate from the dynamic instability associated with single protective barrier crossings. This is, of course, a simplification and yet the assumption could naturally explain why mortality and the incidence of major age-related diseases increase exponentially with age at approximately the same rate [3].

The reduction of slow organism state dynamics to that of a single variable is typical for the proximity of a tipping or critical point [27]. DMI is therefore the property of the organism as a whole, rather than a characteristics of any specific functional subsystem or organism compartment. Indeed, the associations of individual CBC components with the DMI extend over the functional clustering shown in Fig. 1A. In the vicinity of a critical point, fluctuations associated with the criticality become amplified and hence the DMI should be identifiable with the signal components explaining most of the variance in virtually every biological signal (see, e.g., 7, 43, for reviews including performance assessment of variance-based biological age models (e.g. based on Principal Component Analysis). In agreement with the proposed qualitative picture, close to the critical point, the inverse variance of the apparently unrelated physiological variable, the logtransformed physical activity, vanishes at approximately the same age as that of the blood-derived markers. In our recent study [12], we observed that the first princi- 
pal component score in the configuration space spanning the physical activity acceleration/deceleration statistics was strongly associated with mortality, incidence of first morbidity, and health status.

According to the presented model, external stresses (such as smoking) or diseases produce perturbations that modify the shape of the effective potential leading to the shift of the equilibrium DMI position. For example, the mean DMI values in cohorts of individuals who never smoked or who quit smoking are indistinguishable from each other, yet significantly different from (lower than) the mean DMI in the cohort of smokers (Fig. 2C). Thus, the effect of the external stress factor is reflected by a change in the DMI and is reversed as soon as the factor is removed. These findings agree with earlier observations suggesting that the effects of smoking on remaining lifespan and on the risks of developing diseases are mostly reversible once smoking is ceased well before the onset of chronic diseases [12, 22]. The decline in the lung cancer risk after smoking ablation [44] is slower than the recovery rate reported here. This may be the evidence suggesting that long-time stresses may cause hard-to repair damage to the specific tissues and thus produce lasting effects on the resilience.

The reversible character of the DMI deviations in healthy subjects suggests a complex relationship between the DMI and survival. On the one hand, the elevation of physiological variables associated with the DMI indicates reversible activation of the most generic protective stress responses at younger ages, when the organism state is dynamically stable. Moderately elevated DMI levels are therefore a marker of generic stress that can measured by molecular markers (e.g., C-reactive protein) and affects general physical and mental health status [26]. On the other hand, the excessive DMI observed in older individuals can be thought of as an aberrant activation of stress-responses beyond the dynamic stability range. This is a characteristics of chronic diseases and death.

We propose that therapies targeting frailty-associated phenotypes (e.g., inflammation) would, therefore, produce distinctly different effects in disease-free versus frail populations. In healthy subjects, who reside in the region of the stability basin $(B)$ (see Fig. 3D), a treatmentinduced reduction of DMI would quickly saturate over the characteristic auto-correlation time and lead to a moderate decrease in long-term risk of morbidity and death without a change in resilience. Technically, this would translate into an increase in healthspan, although the reduction of health risks would be transient and disappear after cessation of the treatment. In frail individuals, however, the intervention could produce lasting effects and reduce frailty, thus increasing lifespan beyond healthspan. This argument may be supported by longitudinal studies in mice suggesting that the organism state is dynamically unstable, the organism state fluctuations get amplified exponentially at a rate compatible with the mortality rate doubling time, and the effects of transient treatments with life-extending drugs such as rapamycin produce a lasting attenuation of frailty index [45].

The emergence of chronic diseases out of increasingly unstable fluctuations of the organism state provides the necessary dynamic argument to support the derivation of the Gompertz mortality law in the Strehler-Mildvan theory of aging [46]. In [47, 48, the authors suggested that the exponential growth of disease burden observed in the National Population Health Survey of Canadians over 20 y.o. could be explained by an age-related decrease in organism recovery in the face of a constant rate of exposure to environmental stresses. Our study provides evidence suggesting that vanishing resilience cannot be avoided even in the most successfully aging individuals and, therefore, could explain the very high mortality seen in cohorts of super-centennials characterized by the socalled compression of morbidity (late onset of age-related diseases [49]). Formally, such a state of "zero-resilience" at the critical point corresponds to the absolute zero on the vitality scale in the Strehler-Mildvan theory of aging, thus representing a natural limit on human lifespan

The semi-quantitative description of human aging and morbidity proposed here belongs to a class of phenomenological models. Whereas it is possible to associate the variation of the organism state measured by DMI with the effects of stresses or diseases, the data analysis presented here does not provide any mechanistic explanations for the progressive loss of resilience. It is worth to note that the recent study predicts the maximum human lifespan limit from telomere shortening [50] that is compatible with the estimations presented here. It would therefore be interesting to see if the resilience loss in human cohorts is associated or even caused by the loss of regenerative capacity due to Hayflick limit.

The proximity to the critical point indicates that the apparent human lifespan limit is not likely to be improved by therapies aimed against specific chronic diseases or frailty syndrome. Thus, no strong life extension is possible by preventing or curing diseases without interception of the aging process, the root cause of the underlying loss of resilience. We do not foresee any laws of nature prohibiting such an intervention. Therefore, further development of the aging model presented in this work has may eventually lead to experimental demonstration of a dramatic life-extending therapy.

\section{MATERIALS AND METHODS}

Full details for the materials and methods used in this study, including information of the CBC parameters, Cox proportional hazards model, health conditions, and analysis of physiological state fluctuations are provided in the Supplementary Information Appendix. 
[1] Arnold Mitnitski and Kenneth Rockwood. The rate of aging: the rate of deficit accumulation does not change over the adult life span. Biogerontology, 17(1):199-204, 2016.

[2] Ruby Yu, Wan-Chi Wu, Jason Leung, Susan C Hu, and Jean Woo. Frailty and its contributory factors in older adults: a comparison of two asian regions (hong kong and taiwan). International journal of environmental research and public health, 14(10):1096, 2017.

[3] Aleksandr Zenin, Yakov Tsepilov, Sodbo Sharapov, Evgeny Getmantsev, Leonid Menshikov, Peter Fedichev, and Yurii Aulchenko. Identification of 12 genetic loci associated with human healthspan. bioRxiv, page 300889, 2018.

[4] Dmitriy I Podolskiy, Alexei V Lobanov, Gregory V Kryukov, and Vadim N Gladyshev. Analysis of cancer genomes reveals basic features of human aging and its role in cancer development. Nature communications, $7: 12157,2016$.

[5] Teresa Niccoli and Linda Partridge. Ageing as a risk factor for disease. Current Biology, 22(17):R741-R752, 2012.

[6] Nir Barzilai and Gad Rennert. The rationale for delaying aging and the prevention of age-related diseases. Rambam Maimonides medical journal, 3(4), 2012.

[7] M. E. Levine. Modeling the rate of senescence: can estimated biological age predict mortality more accurately than chronological age? J. Gerontol. A Biol. Sci. Med. Sci., 68(6):667-674, Jun 2013.

[8] G. Hannum, J. Guinney, L. Zhao, L. Zhang, G. Hughes, S. Sadda, B. Klotzle, M. Bibikova, J. B. Fan, Y. Gao, R. Deconde, M. Chen, I. Rajapakse, S. Friend, T. Ideker, and K. Zhang. Genome-wide methylation profiles reveal quantitative views of human aging rates. Mol. Cell, 49(2):359-367, Jan 2013.

[9] S. Horvath. DNA methylation age of human tissues and cell types. Genome Biol., 14(10):R115, 2013.

[10] Philippe Terrier and Fabienne Reynard. Effect of age on the variability and stability of gait: a cross-sectional treadmill study in healthy individuals between 20 and 69 years of age. Gait $\&$ posture, 41(1):170-174, 2015.

[11] Tim Althoff, Jennifer L Hicks, Abby C King, Scott L Delp, Jure Leskovec, et al. Large-scale physical activity data reveal worldwide activity inequality. Nature, 547(7663):336, 2017.

[12] Timothy V. Pyrkov, Evgeny Getmantsev, Boris Zhurov, Konstantin Avchaciov, Mikhail Pyatnitskiy, Leonid Menshikov, Kristina Khodova, Andrei V. Gudkov, and Peter O. Fedichev. Quantitative characterization of biological age and frailty based on locomotor activity records. Aging, 10(10):2973-2990, 2018.

[13] Carlos López-Otín, Maria A Blasco, Linda Partridge, Manuel Serrano, and Guido Kroemer. The hallmarks of aging. Cell, 153(6):1194-1217, 2013.

[14] Zuyun Liu, Pei-Lun Kuo, Steve Horvath, Eileen Crimmins, Luigi Ferrucci, and Morgan Levine. Phenotypic age: a novel signature of mortality and morbidity risk. bioRxiv, page 363291, 2018.

[15] Timothy V Pyrkov, Konstantin Slipensky, Mikhail Barg, Alexey Kondrashin, Boris Zhurov, Alexander Zenin, Mikhail Pyatnitskiy, Leonid Menshikov, Sergei Markov, and Peter O Fedichev. Extracting biological age from biomedical data via deep learning: too much of a good thing? Scientific reports, 8(1):5210, 2018.

[16] David R Cox. Regression models and life-tables. In Breakthroughs in statistics, pages 527-541. Springer, 1992.

[17] K Rockwood, JM Blodgett, O Theou, MH Sun, HA Feridooni, A Mitnitski, RA Rose, J Godin, E Gregson, and SE Howlett. A frailty index based on deficit accumulation quantifies mortality risk in humans and in mice. Scientific Reports, 7, 2017.

[18] Benjamin Gompertz. On the nature of the function expressive of the law of human mortality, and on a new mode of determining the value of life contingencies. Philosophical transactions of the Royal Society of London, 115:513-583, 1825.

[19] Andrea Ganna and Erik Ingelsson. 5 year mortality predictors in 498103 uk biobank participants: a prospective population-based study. The Lancet, 386(9993):533-540, 2015.

[20] R OâĂŹdonnell, D Breen, S Wilson, and R Djukanovic. Inflammatory cells in the airways in copd. Thorax, 61(5):448-454, 2006.

[21] Tariq Bhat, Sumaya Teli, Jharendra Rijal, Hilal Bhat, Muhammad Raza, Georges Khoueiry, Mustafain Meghani, Muhammad Akhtar, and Thomas Costantino. Neutrophil to lymphocyte ratio and cardiovascular diseases: a review. Expert review of cardiovascular therapy, 11(1):55-59, 2013.

[22] Donald H Taylor Jr, Vic Hasselblad, S Jane Henley, Michael J Thun, and Frank A Sloan. Benefits of smoking cessation for longevity. American journal of public health, 92(6):990-996, 2002.

[23] LD Landau and EM Lifshitz. Physical kinetics, vol. 10. Course of Theoretical Physics, 1981.

[24] Gregory Hicks and Ram R Miller. Physiological resilience. In Resilience in Aging, pages 89-103. Springer, 2011.

[25] N Jennifer Klinedinst and Alisha Hackney. Physiological resilience and the impact on health. In Resilience in Aging, pages 105-131. Springer, 2018.

[26] Timothy V Pyrkov and Peter O Fedichev. Biological age is a universal marker of aging, stress, and frailty. bioRxiv, page 578245, 2019.

[27] Marten Scheffer, Jordi Bascompte, William A Brock, Victor Brovkin, Stephen R Carpenter, Vasilis Dakos, Hermann Held, Egbert H Van Nes, Max Rietkerk, and George Sugihara. Early-warning signals for critical transitions. Nature, 461(7260):53-59, 2009.

[28] Yonguk Kim, Eung Suk Kim, Seung-Young Yu, and Hyung Woo Kwak. Age-related clinical outcome after macular hole surgery. Retina, 37(1):80-87, 2017.

[29] Jana M Mossey, Elizabeth Mutran, Kathryn Knott, and Rebecca Craik. Determinants of recovery 12 months after hip fracture: the importance of psychosocial factors. American journal of public health, 79(3):279-286, 1989.

[30] Kenneth J Koval, Mary Louise Skovron, Gina B Aharonoff, and Joseph D Zuckerman. Predictors of functional recovery after hip fracture in the elderly. Clinical orthopaedics and related research, 1(348):22-28, 1998. 
[31] NT Artinian, C Duggan, and P Miller. Age differences in patient recovery patterns following coronary artery bypass surgery. American Journal of Critical Care, 2(6):453-461, 1993.

[32] Jacqueline Yewande Thompson, Christopher Byrne, Mark A Williams, David J Keene, Micheal Maia Schlussel, and Sarah E Lamb. Prognostic factors for recovery following acute lateral ankle ligament sprain: a systematic review. BMC musculoskeletal disorders, 18(1):421, 2017.

[33] Hagai Yanai, Arie Budovsky, Robi Tacutu, and Vadim E Fraifeld. Is rate of skin wound healing associated with aging or longevity phenotype? Biogerontology, 12(6):591$597,2011$.

[34] Svetlana Ukraintseva, Anatoliy I Yashin, and Konstantin G Arbeev. Resilience versus robustness in aging, 2016.

[35] Olde Rikkert, GM Marcel, Vasilis Dakos, Timothy G Buchman, Rob de Boer, Leon Glass, Angélique OJ Cramer, Simon Levin, Egbert van Nes, George Sugihara, et al. Slowing down of recovery as generic risk marker for acute severity transitions in chronic diseases. Critical care medicine, 44(3):601-606, 2016.

[36] Marten Scheffer. Complex systems: foreseeing tipping points. Nature, 467(7314):411, 2010.

[37] Sanne MW Gijzel, Heather E Whitson, Ingrid A van de Leemput, Marten Scheffer, Dieneke van Asselt, Jerrald L Rector, Marcel GM Olde Rikkert, and René JF Melis. Resilience in clinical care: Getting a grip on the recovery potential of older adults. Journal of the American Geriatrics Society, 2019.

[38] Heather E Whitson, Wei Duan-Porter, Kenneth E Schmader, Miriam C Morey, Harvey J Cohen, and Cathleen S Colón-Emeric. Physical resilience in older adults: systematic review and development of an emerging construct. Journals of Gerontology Series A: Biomedical Sciences and Medical Sciences, 71(4):489-495, 2015.

[39] Thomas R Dawber, Gilcin F Meadors, and Felix E Moore Jr. Epidemiological approaches to heart disease: the framingham study. American Journal of Public Health and the Nations Health, 41(3):279-286, 1951.

[40] Daniel W Belsky, Avshalom Caspi, Renate Houts, Harvey J Cohen, David L Corcoran, Andrea Danese, HonaLee Harrington, Salomon Israel, Morgan E Levine, Jonathan D Schaefer, et al. Quantification of biological aging in young adults. Proceedings of the National Academy of Sciences, 112(30):E4104-E4110, 2015.

[41] Ayelet Alpert, Yishai Pickman, Michael Leipold, Yael Rosenberg-Hasson, Xuhuai Ji, Renaud Gaujoux, Hadas Rabani, Elina Starosvetsky, Ksenya Kveler, Steven Schaffert, et al. A clinically meaningful metric of immune age derived from high-dimensional longitudinal monitoring. Nature medicine, 25(3):487, 2019.

[42] Sara Ahadi, Wenyu Zhou, Sophia Miryam SchüsslerFiorenza Rose, M Reza Sailani, Kévin Contrepois, Monika Avina, Melanie Ashland, Anne Brunet, and Michael Snyder. Personal aging markers and ageotypes revealed by deep longitudinal profiling. Nature Medicine, 26(1):83-90, 2020.

[43] Linpei Jia, Weiguang Zhang, Rufu Jia, Hongliang Zhang, and Xiangmei Chen. Construction formula of biological age using the principal component analysis. BioMed research international, 2016, 2016.
[44] Hilary A Tindle, Meredith Stevenson Duncan, Robert A Greevy, Ramachandran S Vasan, Suman Kundu, Pierre P Massion, and Matthew S Freiberg. Lifetime smoking history and risk of lung cancer: Results from the framingham heart study. JNCI: Journal of the National Cancer Institute, 110(11):1201-1207, 2018.

[45] Konstantin Avchaciov, Marina P Antoch, Ekaterina L Andrianova, Andrei E Tarkhov, Leonid I Menshikov, Andrei V Gudkov, and Peter O Fedichev. Identification of a blood test-based biomarker of aging through deep learning of aging trajectories in large phenotypic datasets of mice. bioRxiv, 2020.

[46] Bernard L Strehler and Albert S Mildvan. General theory of mortality and aging. Science See Saiensu, 132, 1960.

[47] Arnold Mitnitski, Xiaowei Song, and Kenneth Rockwood. Assessing biological aging: the origin of deficit accumulation. Biogerontology, 14(6):709-717, 2013.

[48] Arnold Mitnitski and Kenneth Rockwood. Aging as a process of deficit accumulation: its utility and origin. In Aging and Health-A Systems Biology Perspective, volume 40, pages 85-98. Karger Publishers, 2015.

[49] Stacy L Andersen, Paola Sebastiani, Daniel A Dworkis, Lori Feldman, and Thomas T Perls. Health span approximates life span among many supercentenarians: compression of morbidity at the approximate limit of life span. Journals of Gerontology Series A: Biomedical Sciences and Medical Sciences, 67(4):395-405, 2012.

[50] Kurt Whittemore, Elsa Vera, Eva Martínez-Nevado, Carola Sanpera, and Maria A Blasco. Telomere shortening rate predicts species life span. Proceedings of the National Academy of Sciences, 116(30):15122-15127, 2019. 


\section{SUPPLEMENTARY INFORMATION}

\section{Complete Blood Count Datasets}

NHANES CBC data were retrieved from the category "Complete Blood Count with 5-part Differential - Whole Blood" for NHANES surveys 1999 - 2010. Corresponding UKB CBC data fields with related database codes are listed in Table $\mathrm{S} 1$. Samples with missing (or filled with zero) data for any of the used CBC components were discarded. Differential white blood cell percentages were converted to cell counts by multiplication by $0.01 \times$ WBC. Neutrophils $(\mathrm{NEU})$ data field was calculated as $0.01 \times \mathrm{WBC} \times(100 \%-\mathrm{MONO}-\mathrm{LYM}-\mathrm{EOS})$ (thus actually included neutrophils and basophils). After these calculations, all CBC parameters were logtransformed.

\section{Hazards model}

The Cox proportional hazards model was trained using NHANES 2015 Public-Use Linked Mortality data. CBC data and mortality linked follow-up available for 40592 NHANES participants aged $18-85$ y.o. was used. Cox model was trained based on data of participants aged 40 - 85 y.o. (11731 male and 12076 female) with 3792 recorded death events during follow-up until the year 2015 (1999 - 2014 surveys). CBC components and the biological sex label were used as covariates. The model was well-predictive of all-cause mortality and yielded a concordance index value of $\mathrm{CI}=0.68$ in NHANES and $\mathrm{CI}=0.66$ in UKB (samples collected 2007-2011, 216250 male and 255223 female participants aged $39-75$ y.o., 13162 recorded death events during follow-up until the year 2016). The Cox proportional hazards model was used as implemented in lifelines package (version 0.14.6) in python. The model was then applied to calculate the hazards ratio for all samples in the GEROLONG, UKB and NHANES cohorts (including individuals younger than 40 y.o.).

\section{The most prevalent chronic diseases and health status}

We quantified the health status of individuals using the sum of major age-related medical conditions that they were diagnosed with, which we termed the compound morbidity index, CMI. The CMI is similar in spirit to the frailty index suggested for NHANES [17]. We were not able to use the frailty index because it was based on Questionnaire and Examination data that were not consistent between all NHANES surveys. Also, we did not have enough corresponding data for the UKB dataset. For CMI determination, we followed [49] and selected the top 11 morbidities strongly associated with age after the age of 40. The list of health conditions included cancer (any kind), cardiovascular conditions (angina pectoris, coronary heart disease, heart attack, heart failure, stroke, or hypertension), diabetes, arthritis and emphysema. Notably, we did not include dementia in the list of diseases since it occurs late in life and hence is severely underrepresented in the UKB cohort due to its limited age range. We categorized participants who had more than 6 of those conditions as the "most frail" (CMI >0.6), and those with CMI $<0.1$ as the "non-frail". NHANES data for diagnosis with a health condition and age at diagnosis is available in the questionnaire category "Medical Conditions" (MCQ). Data on diabetes and hypertension was retrieved additionally from questionnaire categories "Diabetes" (DIQ) and "Blood Pressure \& Cholesterol" (BPQ), respectively.

UK Biobank does not provide aggregated data on these medical conditions. Rather, it provides self-reported questionnaire data (UKB, Category 100074) and diagnoses made during hospital in-patient stay according to ICD10 codes (UKB, Category 2002). We aggregated selfreported and ICD10 (block level) data to match that of NHANES for transferability of the results between populations and datasets. We used the following ICD10 codes to cover the health conditions in UK Biobank: hypertension (I10-I15), arthritis (M00-M25), cancer (C00C99), diabetes (E10-E14), coronary heart disease (I20I25), myocardial infarction (I21, I22), angina pectoris (I20), stroke (I60-I64), emphysema (J43, J44), and congestive heart failure (I50).

\section{Analysis of physiological state fluctuations}

The data presented in this manuscript suggests that DMI fluctuations are governed by external factors as well as intrinsic forces that can be restorative or disruptive. The fluctuations in DMI are fast compared to the rate of aging changes and, therefore, the dynamics of the organism state can be described by the stochastic Langevine equation

$$
\dot{x}(t)=-\varepsilon x(t)+f(t),
$$

here $\varepsilon>0$, and $x(t)=\mathrm{DMI}(t)-\mathrm{DMI}_{0}$ is the deviation of the DMI from its equilibrium value $\mathrm{DMI}_{0}$, determined by the individual organism, environment and life history properties. The recovery rate $\varepsilon$ is proportional to inverse equilibration time and characterizes deterministic forces responsible for the organism state maintenance. The inevitable stochastic factors, $f(t)$, stand for the effects of endogenous and external stress, are assumed zero-mean and uncorrelated, $\left\langle f(t) f\left(t^{\prime}\right)\right\rangle=2 B \delta\left(t-t^{\prime}\right)$ with $B$ being the power of the stochastic stress $(\langle\ldots\rangle$ stands for the averaging over realization of the random process).

The stationary solution of Eq. (1) is a random function with zero mean and variance $\sigma^{2}=\left\langle\delta x^{2}\right\rangle=B / \varepsilon$. That is why we choose to plot the recovery rate from the auto-correlation function and the inverse DMI fluctuations variance $1 / \sigma^{2}$ and in Figs. $3 \mathrm{~A}$ and $3 \mathrm{C}$, respectively. 
bioRxiv preprint doi: https://doi.org/10.1101/618876; this version posted February 7, 2020. The copyright holder for this preprint (which was not certified by peer review) is the author/funder. All rights reserved. No reuse allowed without permission.

To obtain an estimate for the recovery rate $\varepsilon$, we used the normalized fluctuations as a function of the time lag $\Delta t$ between the measurements,

$$
\left\langle(x(t+\Delta t)-x(t))^{2}\right\rangle_{t} / 2 \sigma^{2}=\left(1-e^{-\varepsilon \Delta t}\right) .
$$

Here $\langle\ldots\rangle_{t}$ stands for the everaging along the individual trajectories. 
bioRxiv preprint doi: https://doi.org/10.1101/618876; this version posted February 7, 2020. The copyright holder for this preprint (which was not certified by peer review) is the author/funder. All rights reserved. No reuse allowed without permission.

TABLE S1: CBC data used in the study.

\begin{tabular}{llll} 
CBC component & Abbreviation & NHANES & UKB \\
\hline Hemoglobin & HB & LBXHGB & Haemoglobin concentration (30020) \\
Red blood cell count & RBC & LBXRBCSI & Red blood cell (erythrocyte) count (20010) \\
Mean corpuscular volume & MCV & LBXMCVSI & Mean corpuscular volume (30040) \\
Mean corpuscular hemoglobin concentration & MCHC & LBXMC & Mean corpuscular haemoglobin concentration (30060) \\
Red blood cell distribution width & RDW & LBXRDW & Red blood cell (erythrocyte) distribution width (30070) \\
Platelets & PLT & LBXPLTSI & Platelet count (30080) \\
Monocytes, \% & MONO & LBXMOPCT Monocyte percentage (30190) \\
Lymphocytes, \% & LYM & LBXLYPCT Lymphocyte percentage (30180) \\
Eosinophils, \% & EOS & LBXEOPCT Eosinophill percentage (30210) \\
White blood cell count & WBC & LBXWBCSI White blood cell (leukocyte) count (30000)
\end{tabular}

TABLE S2: Significance of prediction of acquiring a health condition based on estimated log hazards ratio (adjusted for age and gender). Only UKB subjects with none of the listed health conditions at the time of survey were considered; the total number of subjects evaluated for each condition was 263956. The numbers in parentheses in the far right column indicate the occurrence of the disease being the first diagnosis in an individual.

\begin{tabular}{llll} 
Condition & HR $(95 \%$ CI $)$ & p-value & $\mathrm{n}_{\text {events }}\left(\mathrm{n}_{\text {is first morbidity }}\right)$ \\
\hline Death & $1.35(1.33-1.37)$ & $4.9 \mathrm{E}-110$ & $4745(927)$ \\
First morbidity & $1.05(1.05-1.06)$ & $3 \mathrm{E}-40$ & $68126(68126)$ \\
Hypertension & $1.04(1.04-1.05)$ & $1.2 \mathrm{E}-13$ & $31143(25681)$ \\
Arthritis & $1.07(1.07-1.08)$ & $1.7 \mathrm{E}-32$ & $28745(24451)$ \\
cancers & $1.03(1.02-1.04)$ & $2.7 \mathrm{E}-05$ & $18838(15860)$ \\
Coronary heart disease & $1.05(1.04-1.06)$ & $2.9 \mathrm{E}-05$ & $7422(5500)$ \\
Diabetes & $1.03(1.02-1.05)$ & 0.0084 & $6605(5265)$ \\
Angina pectoris & $1.02(1.00-1.03)$ & 0.35 & $3747(2164)$ \\
Emphysema & $1.48(1.45-1.51)$ & $4.5 \mathrm{E}-108$ & $2382(1508)$ \\
Heart attack & $1.05(1.03-1.08)$ & 0.012 & $2186(1605)$ \\
Stroke & $1.10(1.08-1.13)$ & $4.1 \mathrm{E}-05$ & $1686(1168)$ \\
Heart failure & $1.32(1.29-1.36)$ & $6.2 \mathrm{E}-26$ & $1209(583)$ \\
Bronchitis & $1.18(1.12-1.25)$ & 0.0034 & $280(177)$
\end{tabular}
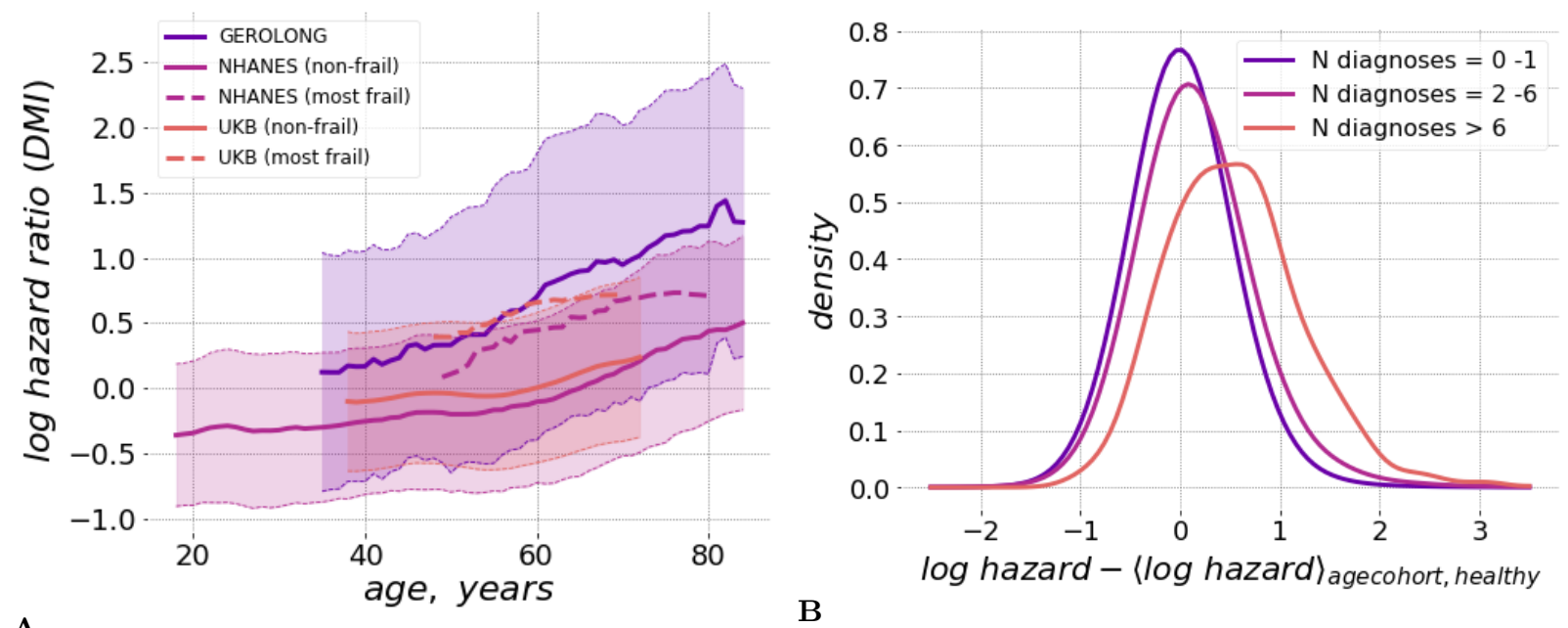

A

B

FIG. S1: A. DMI mean values (lines) and variance (shaded areas) are plotted relative to age for the NHANES (same as in Fig. 1B), UKB and GEROLONG datasets (color-matched with respect to each study). For NHANES and UKB the solid line and shaded regions mark the population average ad the range spanned by one standard deviation from it for the "non-frail" (CMI < 0.1) participats. The population mean for the "most frail" (CMI > 0.6) individuals is shown with dashed lines. B. Distributions of sex- and age-adjusted DMI in cohorts of UKB participants in different morbidity categories relative to the DMI mean in cohorts of "non-frail" (one or no diagnoses, CMI $<0.1$ ) individuals. Note that the distribution function in the "most frail" group (more than 6 diagnoses, CMI $>0.6$ ) exhibited the largest shift and a profound deviation from the symmetric form, similarly as it was seen in NHANES. 